\title{
Minimal Volume Simplex (MVS) Approach for Convex Hull Generation in TP Model Transformation
}

\author{
József Kuti*, Péter Galambos*†, Péter Baranyi* \\ *Institute for Computer Science and Control, Hungarian Academy of Sciences \\ Kende u. 13-17. H-1111 Budapest, Hungary \\ ${ }^{\dagger}$ Antal Bejczy Center for Intelligent Robotics, Óbuda University, \\ Bécsi út 96/B. H-1034 Budapest, Hungary \\ E-mail: kuti,galambos, baranyi@sztaki.mta.hu
}

\begin{abstract}
To a large degree, systems and control applications of TP Model Transformation rely on convex hull manipulation of polytopic LPV/qLPV system models. In this respect, the creation of tight convex hulls is an especially challenging problem, as it requires complex nonlinear optimisation. By defining the Minimal Volume Simplex (MVS) type hull, the paper presents a novel approach for tight convex hull generation. The approach, which involves the so-called MVSA algorithm, leads to a radical reduction in computational time while showing improved numerical properties in terms of repeatability and reliability as compared to other hull generation methods. Furthermore, the proposed method allows for the taking into account of special design considerations regarding the alignment of the convex hull.

Index Terms-Systems and Control, TP Model Transformation, Polytopic LPV/qLPV modelling, Convex hull generation
\end{abstract}

\section{INTRODUCTION}

In the past decade, TP Model Transformation has proved its efficiency in various applications [1], [2]. The power of the method lies in its gateway role since it delivers the convex polytopic representation of quasi Linear Parameter Varying (LPV/qLPV) system models that is directly serves as input for multi-objective Linear Matrix Inequality (LMI) based control design. The tight convex hull is vital in control synthesis since LMI-based methods are very sensitive to the hull's alignment and its volume. As a rule of thumb, it can be said that the smaller hull we have, the more likely be the LMI problem feasible (or better performance metrics can be reached).

In the context of TP Model Transformation, the tight convex hull is usually generated by the so-called Close NOrmalisation (CNO) method [3]. This approach interprets the tightness as the distance of the hull and the actual model via a heuristic closeness function, then utilizes it as cost function in the optimization based on random search combined with the commonly used Nelder-Mead nonlinear optimisation [4]. Unfortunately, over a certain model complexity this method is very time-consuming and often get stuck in local minima very far from the optimum.

In this paper, we introduce the Minimal Volume Simplex (MVS) approach to determine the tight hull, where the simplex hull's volume is minimized. At the level of underlying mathematics, a similar problem appears in material classification and recognition from hyperspectral images [5]. Some remarkable results on hyperspectral unmixing are the Alternating Projected Subgradients [6], the Non-negative Least-correlated Component Analysis (nLCA) [7], the Non-negative Matrix Factorization Minimum Volume Transform (NMF-MVT) [8], the Minimal Volume Simplex Analysis (MVSA) [9] and the Minimal Volume Enclosed Simplex (MVES) [10]. The MVSA algorithm is outstanding in terms of computational time and efficiency [10], [11].

Based on the MVSA, the proposed Minimal Volume Simplex (MVS) hull generation approach gives a fast and numerically reliable (i.e., consecutive executions give identical result) method for preparing the tight convex hull of polytopic LPV/qLPV models of dynamical systems.

The paper is structured as follows: Notations are introduced in section II, then section III gives a brief introduction to the TP Model Transformation. In section IV-A, the main steps of MVS hull generation are shown based on the MVSA algorithm while in section IV-B, a modified method presented applying special additional constraints. Section V presents numerical examples to give practical insight into the application of the method and to compare the MVS and CNO approaches. Finally, Section VI concludes the paper.

\section{NOTATIONS}

The following notations are used within this paper:

$a, b, \ldots \quad$ scalar values

$\mathbf{a}, \mathbf{b}, \ldots \quad$ vectors

$\mathbf{A}, \mathbf{B}, \ldots$ matrices

$\mathbf{A} \otimes \mathbf{B} \quad$ Kronecker product

$\mathbf{0}^{a \times b}, \mathbf{1}^{a \times b} \quad a \times b$ size matrix of zeros/ones

$\mathbf{E}^{a \times b} \quad a \times b$ size identify matrix

$\mathcal{A}, \mathcal{B}, \ldots \quad$ tensors

$\mathcal{A} \underset{n=1}{\mathbb{N}} \mathbf{U}_{n} \quad$ multiple tensor product as $\mathcal{A} \times{ }_{1} \mathbf{U}_{1} \cdots \times_{N} \mathbf{U}_{N}$ 


\section{BASIC CONCEPTS}

Consider the following typical LPV/qLPV model:

$$
\dot{\mathbf{x}}(t)=\mathbf{S}(\mathbf{p}(t))\left[\begin{array}{l}
\mathbf{x}(t) \\
\mathbf{u}(t)
\end{array}\right]
$$

where

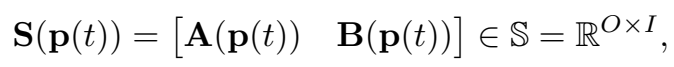

on a hyper-rectangular parameter domain:

$$
\mathbf{p} \in \Omega=\left[p_{1, \min }, p_{1, \max }\right] \times \cdots \times\left[p_{N, \text { min }}, p_{N, \text { max }}\right] \subset \mathbb{R}^{N} .
$$

In the modern control theory, different LMI-based approaches are widely used for stability verification and robust or gain scheduling multi-objective controller design. The TP Model Transformation offers an appealing numerical way to obtain the convex polytopic model of the investigated nonlinear/parameter-dependent system (1) that supports the direct use of LMI-based design methodologies. The TP Model Transformation has two main steps: the determination of the Higher Order SVD (HOSVD) based form and the convex hull manipulation.

The HOSVD-based form of LPV/qLPV models that is introduced by Baranyi in [12], [13] describes the model in a unique tensor product structure.

Definition 1: The following form of (1)

$$
\mathbf{S}(\mathbf{p})=\mathcal{S}^{\text {ortho }} \underset{n=1}{N} \mathbf{u}^{(n)}\left(p_{n}\right) \in \mathbb{S},
$$

is called HOSVD-based form, where if

- the subtensors of $\mathcal{S}^{\text {ortho }} \in \mathbb{S}^{I_{1} \times \cdots \times I_{N}}$ are orthogonal, as

$$
<\mathcal{S}_{i_{n}=j}^{\text {ortho }}, \mathcal{S}_{i_{n}=k}^{\text {ortho }}>=\left\{\begin{array}{cl}
0 & \text { if } j \neq k, \\
\sigma_{j}^{(n)^{2}} & \text { if } j=k,
\end{array}\right.
$$

and ordered: $\sigma_{1}^{(n)} \geq \cdots \geq \sigma_{I_{n}}^{(n)} \geq 0$,

- and the $\mathbf{u}^{(n)}\left(p_{n}\right) \in \mathbb{R}^{1 \times \bar{I}_{n}}$ singular vectors are orthonormal, as

$$
\int_{p_{n}=p_{n}^{\min }}^{p_{n}^{\max }} u_{j}^{(n)}\left(p_{n}\right) u_{k}^{(n)}\left(p_{n}\right) d p_{n}= \begin{cases}0 & \text { if } j \neq k \\ 1 & \text { if } j=k .\end{cases}
$$

This representation is called unique, because the number of the weighting functions and their order is uniquely defined in each dimension respectively (only their signs can vary, and in case of equal singular values they can be underdetermined in the given subspace) [14]. Concerning the convex hull manipulation, the HOSVD-form can be considered as unique.

In geometrical sense, the $n$-mode subtensors of the core tensor $\mathcal{S}^{\text {ortho }}$ form an orthogonal system and the weighting vectors $\mathbf{u}^{(n)}\left(p_{n}\right)$ are coordinates with respect to that basis.

In the rest of the paper, $D_{n}$ denotes the number of the linearly independent scalar functions of the vector function $\mathbf{u}^{(n)}\left(p_{n}\right)-\mathbf{u}_{0}$, where $\mathbf{u}_{0}$ is an arbitrarily chosen value of $\mathbf{u}^{(n)}\left(p_{n}\right)$, which allows for the representation of $\mathbf{u}^{(n)}\left(p_{n}\right)$ by the sum of a constant offset and a vector in a $D_{n}$-dimensional basis.
The orthonormal structure of the HOSVD-based form allows for the separate (decoupled) execution of the convex hull manipulation in each dimensions. The fundamental equation of the convex hull manipulation is formulated by the following lemma.

Lemma 1: If there exist $\mathbf{w}^{(n)}\left(p_{n}\right) \in \mathbb{R}^{1 \times J_{n}},\left(J_{n} \geq D_{n}+1\right)$ convex weighting functions for each $n$ dimensions, that is

$$
\begin{aligned}
& \sum_{i=1}^{J_{n}} w_{i}^{(n)}\left(p_{n}\right)=1, \quad \forall p_{n}, \\
& w_{j}^{(n)}\left(p_{n}\right) \geq 0, \quad \forall j=1 . . J_{n}, p_{n},
\end{aligned}
$$

and there exist $\mathbf{T}^{(n)}$ transformation matrices, such that

$$
\mathbf{w}^{(n)}\left(p_{n}\right) \mathbf{T}^{(n)}=\mathbf{u}^{(n)}\left(p_{n}\right),
$$

the convex TP model can be generally computed as

$$
\begin{aligned}
& \mathbf{S}(\mathbf{p})=\mathcal{S}^{\text {convex }} \underset{n=1}{\stackrel{\otimes}{\otimes}} \mathbf{w}^{(n)}\left(p_{n}\right), \\
& \text { where } \quad \mathcal{S}^{\text {convex }}=\mathcal{S}^{\text {ortho }} \underset{n=1}{N} \mathbf{T}^{(n)} \text {. }
\end{aligned}
$$

In previous works [1], [15], [16] different types of simplex hulls were defined based on the properties of the weighting functions:

- SN: The weighting functions are Sum Normalised (5).

- NN: The weighting functions does not have any negative element (6).

- NO: The weighting functions are SN and NN, and the largest value of each function is 1 .

- CNO: The weighting functions are SN and NN, and the largest value of each function is 1 or ,close" to 1 .

- RNO: The weighting functions are $\mathrm{SN}$ and NN, and the largest values of each function are the same.

- INO: The weighting functions are $\mathrm{SN}$ and $\mathrm{NN}$, and the smallest value of each function is 0 .

\section{Minimal Volume Simplex (MVS) type convex HULL}

In this section, we present a new approach where the tight hull is defined as a Minimal Volume Simplex. The proposed method supports the formulation of special constraints on the properties of the convex hull (e.g., CNO-like hulls), and thus, it can be considered as a common convex hull manipulation framework.

The volume of a simplex is an easily determinable quantity, but the generation of the minimal volume simplex hull is a multivariate nonlinear optimisation problem, wherein there usually exist several local minima. In general, it is not possible to find the global minimum, however, good sub-optimal solution can be given.

The following subsections discuss the MVS hull generation based on the MVSA algorithm and extends the essential method with additional constraints to consider various control aspects. 


\section{A. Minimal volume convex hull based on MVSA}

Consider the matrix $\mathbf{U} \in \mathbb{R}^{M \times I}$ that contains the $D$ dimensional orthonormal weighting functions $\mathbf{u}(p) \in \mathbb{R}^{1 \times I}$ discretized in $M$ points. The goal is to find the MVS hull and the corresponding convex weighting functions $\mathbf{W}^{M V S} \in$ $\mathbb{R}^{M \times J}$ such that

$$
\begin{aligned}
\sum_{j=1}^{J} w_{m, j} & =1 \quad \forall m=1 . . M, \\
w_{m, j} \geq 0 & \forall m=1 . . M, j=1 . . J
\end{aligned}
$$

for which exists $\mathbf{T} \in \mathbb{R}^{J x I}$ such that $\mathbf{W}^{M V S} \mathbf{T}=\mathbf{U}$.

The algorithm consists of three consecutive stages:

STEP 1 (Reformulation of the weighting functions): First, the $J=D+1$ dimensional orthonormal weighting functions $\mathbf{V} \in \mathbb{R}^{M \times J}$ are determined from $\mathbf{U}$ in such a way that the equation

$$
\mathbf{U}=\mathbf{V} \mathbf{T}_{0}
$$

holds and

$[\mathfrak{U}, \mathfrak{S}, \mathfrak{V}]=\operatorname{SVD}\left(\left[\begin{array}{ll}\mathbf{U} & \mathbf{1}^{M}\end{array}\right]\right), \mathbf{V}=\mathfrak{U}, \mathbf{T}_{0}=\mathfrak{S V}^{T} \mathbf{E}^{(I+1) \times I}$.

As a result, the objective of the optimization is now to find the transformation matrix $\mathbf{R} \in \mathbb{R}^{J \times J}$ for which

$$
\mathbf{W}=\mathbf{V R}^{-1}
$$

is a convex system of weighting functions, and the volume of the hull is minimal, that is

$$
\mathbf{R}=\arg \min _{\mathbf{R}}(\operatorname{det} \mathbf{R}) .
$$

The matrix $\mathbf{R}$ contains the $\mathbf{r}_{j} \in \mathbb{R}^{1 \times J}$ vertices in the space of $\mathbf{V}$ weighting functions:

$$
\mathbf{R}=\left[\begin{array}{c}
\mathbf{r}_{1} \\
\vdots \\
\mathbf{r}_{J}
\end{array}\right]
$$

STEP 2 (Finding the initial guess): The optimization requires an initial convex hull which is similarly oriented as the optimal hull. To obtain it, first we determine the dominant elements $\mathbf{r}_{j}^{\text {dom }}(j=1 . . J)$ of the weighting functions $\mathbf{V}$ using the Vertex Component Analysis (VCA) method [17]. Accordingly, $\left(\mathbf{r}_{1}^{\text {dom }}, \ldots, \mathbf{r}_{J}^{\text {dom }}\right)$ results in the maximal volume inner simplex hull. Based on (12) and (14) the affine weighting functions $\mathbf{W}^{\text {dom }}$ for the dominant elements can be obtained with values between \pm 1 . The initial convex hull can now be determined by expanding (,,inflating”) the maximal inner hull that generally results in a good initial guess.

Vertices of the initial hull are computed as

$$
\begin{aligned}
& \quad \mathbf{r}_{j}^{e x}=\mathbf{r}_{j}^{d o m}+\delta\left(\mathbf{r}_{j}^{\text {dom }}-\mathbf{r}_{\text {mean }}\right), \quad \forall j=1 \ldots J \\
& \text { where } \quad \mathbf{r}_{\text {mean }}=\sum_{j=1}^{J} \mathbf{r}_{j}^{\text {dom }} / J, \quad J \geq \delta \geq 0
\end{aligned}
$$

and $\delta$ is the expansion coefficient. It can be shown that if $\delta=J$, the expanded polytope becomes certainly convex. The initial transformation matrix $\mathbf{R}_{0}$ contains the vectors $\mathbf{r}_{j}^{e x}$ as in (14).

STEP 3 (Iterative optimization): It is shown in [9], that if

$$
\mathbf{X}=\left[\begin{array}{lll}
\mathbf{x}_{1} & \ldots & \mathbf{x}_{J}
\end{array}\right]=\mathbf{R}^{-1}, \quad \mathbf{x}=\left[\begin{array}{c}
\mathbf{x}_{1} \\
\vdots \\
\mathbf{x}_{J}
\end{array}\right],
$$

then the (13) cost function can be replaced with

$$
f(\mathbf{x})=-\log |\operatorname{det} \mathbf{X}|
$$

and the conditions of convexity can be formulated as

$$
\begin{aligned}
\left(\mathbf{E}^{J \times J} \otimes \mathbf{V}\right) \mathbf{x} & \geq \mathbf{0}^{J^{2} \times 1}, \\
\left(\mathbf{1}^{1 \times J} \otimes \mathbf{E}^{J \times J}\right) \mathbf{x} & =\sum_{m=1}^{M} \mathbf{v}_{m} .
\end{aligned}
$$

The key idea of the MVSA method is to approximate the cost function (17) around the $\mathbf{x}_{0}$ initial guess (computed from $\mathbf{R}_{0}$ by (16)) as a quadratic function:

$f(\mathbf{x}) \cong f\left(\mathbf{x}_{0}\right)+\mathbf{f} \cdot\left(\mathbf{x}-\mathbf{x}_{0}\right)+0.5\left(\mathbf{x}-\mathbf{x}_{0}\right)^{T} \mathbf{H}\left(\mathbf{x}-\mathbf{x}_{0}\right)$,

where $\mathbf{f}$ and $\mathbf{H}$ are computed from the values of $\mathbf{R}_{0}$ (according to the $\mathbf{x}_{0}$ initial guess) as

$$
\mathbf{f}=-\left[\begin{array}{lll}
\mathbf{r}_{0,1} & \ldots & \mathbf{r}_{0, J}
\end{array}\right], \quad \mathbf{H}=\operatorname{diag}\left(f_{1}^{2}, f_{2}^{2}, \ldots, f_{J^{2}}^{2}\right) .
$$

The optimization of the quadratic expression (20) with respect to (18) and (19), the convex constraints are efficiently computable and the iterative execution results in good suboptimal solution.

From the resulted $\mathbf{x}$ the matrix $\mathbf{R}$ and the corresponding weighting functions $\mathbf{W}^{M V S}$ can be restored by (16) and (12) respectively.

Using the results, the equation of convex hull manipulation forms as $\mathbf{W}^{M V S} \mathbf{T}=\mathbf{U}$, where $\mathbf{T}=\mathbf{R T}_{0}$.

\section{B. Incorporation of additional constrains}

A great advantage of the MVSA method is that the constraints (18)-(19) can be augmented with further equalities/inequalities formulating specific conditions. The subsection discusses this potential through a practically relevant example .

It is a common experience with LMI-based design techniques, that the feasibility or the achievable performance can be radically fostered by forming a specially aligned convex hull wherein two vertices stands right at the endpoints of the system's trajectory in each parameter dimensions. These terminal points usually means the extremities (the most differing LTI systems) and thus, in experience, it is impractical if the hull ranges over the actual model in these directions. In the former $\mathrm{CNO}$ method it was assured by additional penalty terms in the cost function.

Using the MVSA-based method, the constraints

$$
w_{m=1, j=1} \cong 1, \quad w_{m=M, j=J} \cong 1
$$


can be used in the optimization as

$$
\begin{gathered}
\left(\left[\begin{array}{ll}
1 & \mathbf{0}^{1 \times(J-1)}
\end{array}\right] \otimes \mathbf{v}_{m=1}\right) \mathbf{x} \geq 1-\epsilon, \\
\left(\left[\begin{array}{ll}
\mathbf{0}^{1 \times(J-1)} & 1
\end{array}\right] \otimes \mathbf{v}_{m=M}\right) \mathbf{x} \geq 1-\epsilon,
\end{gathered}
$$

where $\epsilon<10^{-3}$.

Through the dominant elements and the corresponding weighting functions we can check, whether there exists appropriate convex hull with the following criteria:

- the end-points are dominant elements, so

$$
\mathbf{w}_{m=1}^{d o m}=\left[\begin{array}{llll}
1 & 0 & \ldots & 0
\end{array}\right], \quad \mathbf{w}_{m=M}^{d o m}=\left[\begin{array}{llll}
0 & \ldots & 0 & 1
\end{array}\right],
$$

- for all $j=1, J, m=1 . . M$ :

$$
\text { if } w_{m, j}^{\text {dom }}<0: \quad w_{s u m *, m}>-2 w_{m, j}^{d o m},
$$

- for all $j=2 . .(J-1), m=1 . . M$ :

$$
\text { if } w_{m, j}^{d o m}<0: \quad w_{s u m *, m}>0,
$$

where $w_{\text {sum } *, m}=\sum_{j=2}^{J-1} w_{m, j}^{d o m} \quad \forall m=1 . . M$.

Then the following constrained expansion method generates the initial convex hull leaving the terminal vertices unmoved:

- First, expand the $J-2$ free vertices in their $J-2$ dimensional space:

$$
\begin{aligned}
& \mathbf{r}_{j}^{e x}=\mathbf{r}_{j}^{d o m}+\delta_{1}\left(\mathbf{r}_{j}^{d o m}-\mathbf{r}_{\text {mean } *}\right) \quad \forall j=2 . .(J-1), \\
& \text { where } \quad \mathbf{r}_{\text {mean } *}=\sum_{j=2}^{J-1} \mathbf{r}_{j}^{d o m} /(J-2) .
\end{aligned}
$$

- Then project them from the fixed vertices:

$$
\mathbf{r}_{j}^{e x}=\mathbf{r}_{i}^{e x}+\delta_{2}\left(2 \mathbf{r}_{j}^{e x}-\mathbf{r}_{1}^{d o m}-\mathbf{r}_{J}^{d o m}\right) \forall j=2 . .(J-1) .
$$

Using appropriately large $\delta_{1}, \delta_{2}$ values the expanded polytope become convex without moving the fixed vertices resulting a good initial guess.

\section{EXAMPLES}

This section presents numerical examples illustrating the proposed convex hull manipulation method and compares the results with the $\mathrm{CNO}$ algorithm that is implemented in the TPTool [3].

The presented examples clearly show the advantages of the MVSA-based approach in terms of computational time and numerical reliability/repeatability.

\section{A. Example 1}

For the sake of simplicity, consider the univariate nonlinear two dimensional vector function

$$
\mathbf{y}(p)=\left[\begin{array}{l}
y_{1}(p) \\
y_{2}(p)
\end{array}\right]=\left[\begin{array}{c}
p \\
p^{2}+0.5
\end{array}\right]
$$

over the parameter interval $p \in[0.5,4]$.

Performing the TP Model Transformation on this function using the proposed convex hull preparation techniques, we get the hulls displayed in Fig. 1(a) that shows well the effect of the additional constraints. The weighting functions for the nonconvex initial guess is shown in Figure 1(b), while the resulted convex weighting function systems are displayed by Figures 1(c)-1(d).

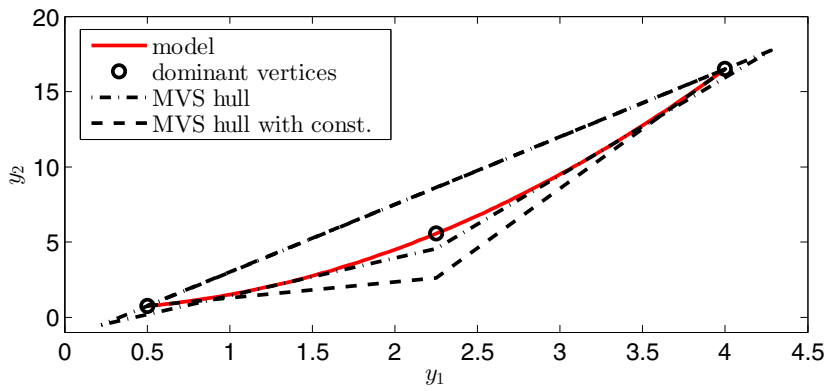

(a) The model, the dominant elements and the MVS convex hulls

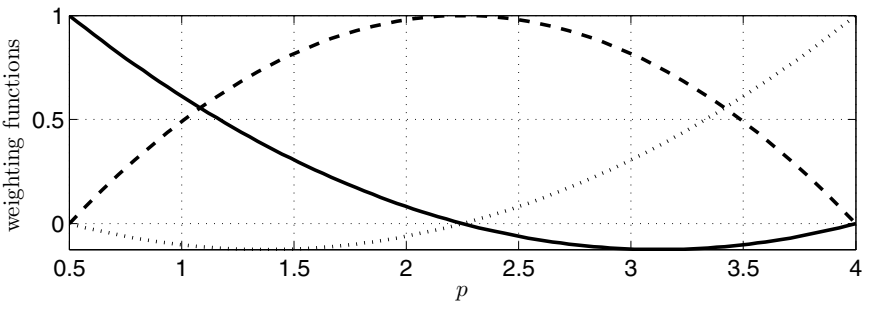

(b) Weighting functions for the dominant elements

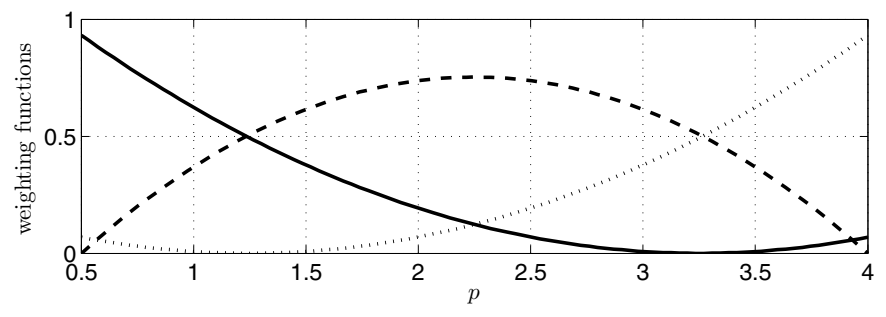

(c) Weighting functions of the Minimal Volume Simplex hull

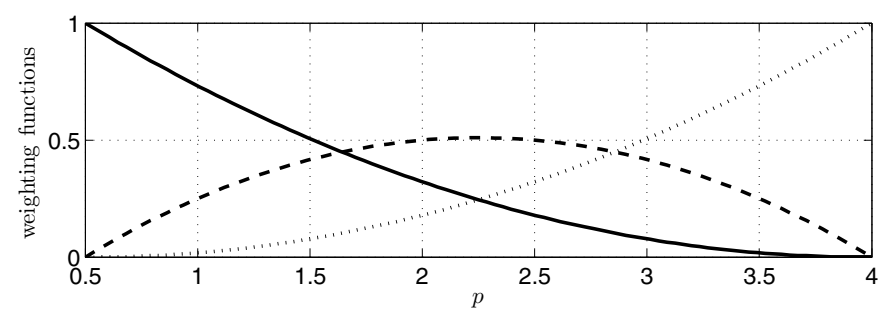

(d) Weighting functions of the MVS hull applying additional constraints

Fig. 1. Results of convex hull manipulation for Example 1

\section{B. Example 2}

Consider the mechanical model of the inverted pendulum (Fig. 2) that can be described in the qLPV state-space form:

$$
\dot{\mathbf{x}}(t)=\mathbf{A}(\mathbf{p}(t)) \mathbf{x}+\mathbf{B}(\mathbf{p}(t)) \mathbf{u}(t),
$$

where

$$
\begin{aligned}
& \mathbf{x}(t)=\left[\begin{array}{llll}
\dot{x} & x & \dot{\alpha} & \alpha
\end{array}\right]^{T}, \quad \mathbf{u}(t)=F, \quad \mathbf{p}(t)=\left[\begin{array}{ll}
|\alpha| & |\dot{\alpha}|
\end{array}\right]^{T}, \\
& \mathbf{A}(\mathbf{p}(t))=\left[\begin{array}{cccc}
0 & 0 & 0 & \frac{m\left(2 p_{2}^{2} l-3 g \cos p_{1}\right)}{D\left(p_{1}\right)} \frac{\sin p_{1}}{p_{1}} \\
1 & 0 & 0 & 0 \\
0 & 0 & 0 & \frac{6 \frac{g}{l}(m+M)-3 m p_{2}^{2} \cos p_{1}}{D\left(p_{1}\right)} \frac{\sin p_{1}}{p_{1}} \\
0 & 0 & 1 & 0
\end{array}\right],
\end{aligned}
$$


$\mathbf{B}(\mathbf{p}(t))=\left[\begin{array}{c}\frac{4}{D\left(p_{1}\right)} \\ 0 \\ \frac{-6 \cos p_{1}}{l \cdot D\left(p_{1}\right)} \\ 0\end{array}\right], \quad D\left(p_{1}\right)=4 M+m\left(1+3 \sin ^{2} p_{1}\right)$.

In the numerical example, the parameter values $m=$ $0.2[\mathrm{~kg}], M=0.5[\mathrm{~kg}], l=0.3[\mathrm{~m}]$ and the parameter domain $p_{1} \in[0,1.48][\mathrm{rad}], p_{2} \in[0,0.087][\mathrm{rad} / \mathrm{s}]$ were considered. The TP Model Transformation was performed on the discretized model that is sampled over a $M_{1}=59$ by $M_{2}=61$ equidistant grid.

In the parameter dimension of $p_{1}$ we got an $I_{1}=5$ element orthonormal weighting function system (Fig. 3(a)), which represents a $D_{1}=4$ dimensional structure. Figure 3(b) shows the non-convex weighting functions for the dominant elements (initial guess), while in Figure 3(c) and 3(d) the different MVSA-based convex weighting function systems are displayed.

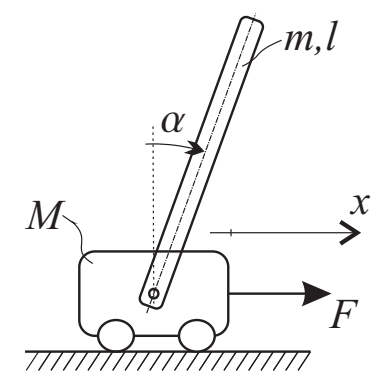

Fig. 2. Inverted pendulum model

Since in this case, the 2D illustration would not be expressive, in the followings we quantify the hulls by their $D=4$ dimensional volume. The volume of the MVSA-based simplex hull is $V_{M V S}=0.0080$ that grows to $V_{M V S *}=0.0106$ applying the additional constraints.

The $I_{2}=2$ element HOSVD-based weighting function system of the $p_{2}$ parameter dimension has a $D_{2}=1$ dimensional structure. Since its minimal volume hull is trivial, the details are not discussed here.

Using the resulted convex polytopic models, we performed the LMI-based quadratic stabilization via static state feedback [18]. In case of the MVS hull, the achievable decay rate is $\alpha=$ $0.023[1 / s]$, while applying the additional constraints (22),(23) the design is feasible up to the decay rate $\alpha=0.099[1 / s]$.

\section{Comparison with the CNO method}

The CNO algorithm implemented in TPTool [3] searches among all possible INO \& SN \& NN hulls by starting the commonly used Nelder-Mead optimization started from random hulls. The cost function depends on the distance between the vertices and the model, and apply special penalty terms to force the vertices to be at the endpoints.

The algorithm is quite efficient for low-dimensional problems, however, if $D>2$ the algorithm is very time-consuming and the consecutive executions lead to very different results.

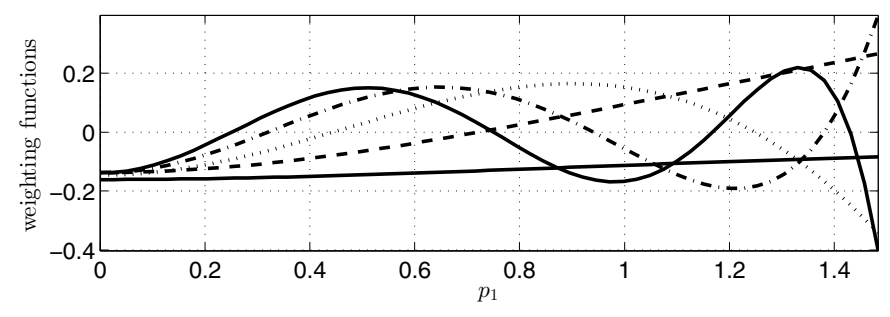

(a) The HOSVD-based orthonormal weighting functions

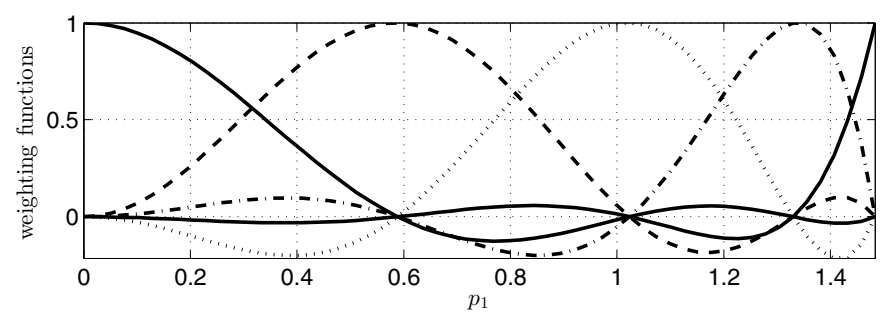

(b) Weighting functions for the dominant elements

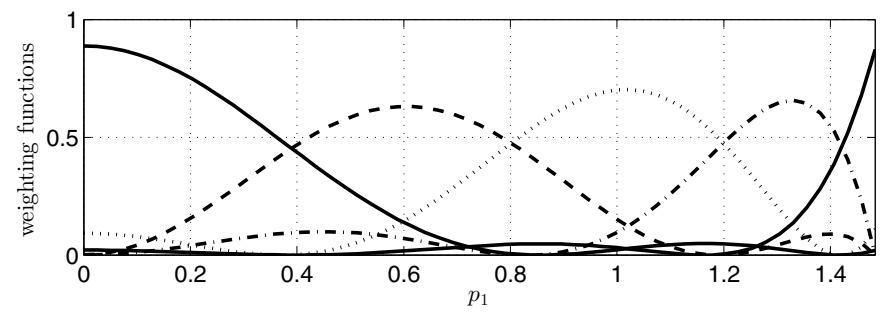

(c) Weighting functions of the MVS hull $\left(V_{M V S}=0.0080\right)$

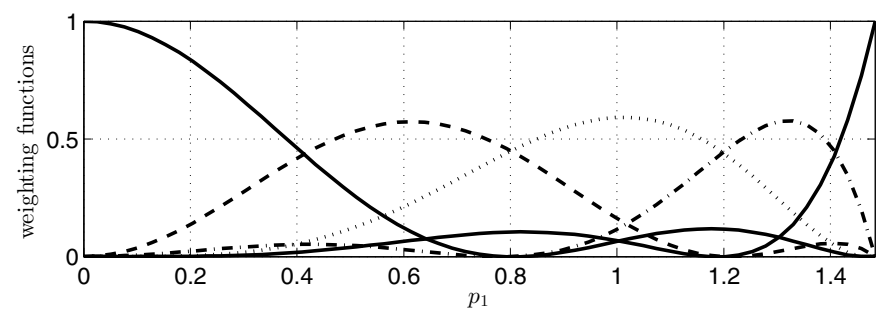

(d) Weighting functions of the MVS hull with additional constraints $\left(V_{M V S *}=0.0106\right)$

Fig. 3. Results of convex hull manipulation for Example 2

Hence, the CNO-type hull is ambiguous as the repeatability of the computation is questionable.

Example 1 is a simple $D=2$ dimensional problem on which the MVSA-based algorithms are much faster. It takes only $0.031[s]$ to find the tight hull using the proposed method, while on the same computer, the CNO method takes $5.41[s]$, which is about 150 times more.

To investigate the "repeatability", the $D=4$ dimensional weighting function system of Example 2 the $\mathrm{CNO}$ and the MVSA-based algorithm were applied 100-100 times. The MVSA-based algorithms resulted consistently the same hull and it takes $\Delta t_{\text {mean }}=0.17[s]$ on average. The CNO method resulted very different hulls (Fig. 4(b),4(c)) and their volume distribution shows a large spread (Fig. 4(a)). One computation takes about 500 times more time: almost $\Delta t_{\text {mean }}=100[s]$ on 


\begin{tabular}{l|c|c|c|c} 
Method & $V_{\text {min }}$ & $V_{\text {mean }}$ & $V_{\text {max }}$ & $\Delta t_{\text {mean }}$ \\
\hline CNO & 0.0098 & 0.0175 & 0.0827 & $98.42[s]$ \\
\hline MVS with constraints & 0.0106 & 0.0106 & 0.0106 & $0.17[s]$ \\
\hline
\end{tabular}

TABLE I

NUMERICAL COMPARISON BASED ON 100-100 EXECUTION

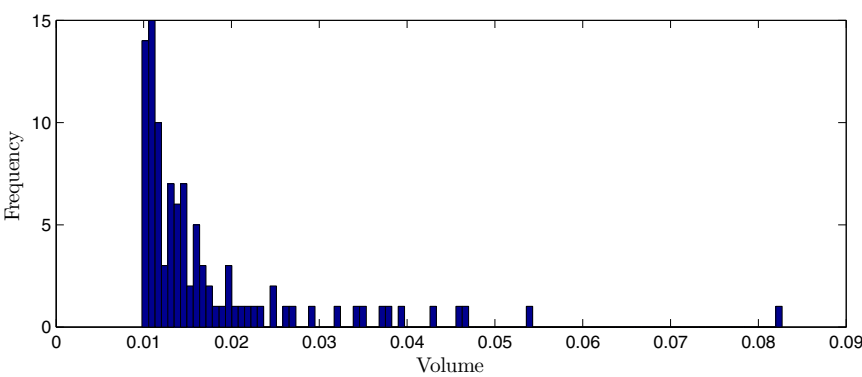

(a) Volume distribution of 100 results of CNO method

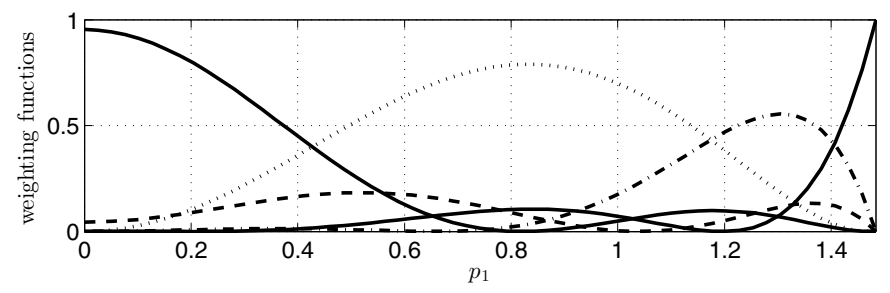

(b) Weighting functions of the CNO convex hull $\left(V_{C N O}=0.0236\right)$

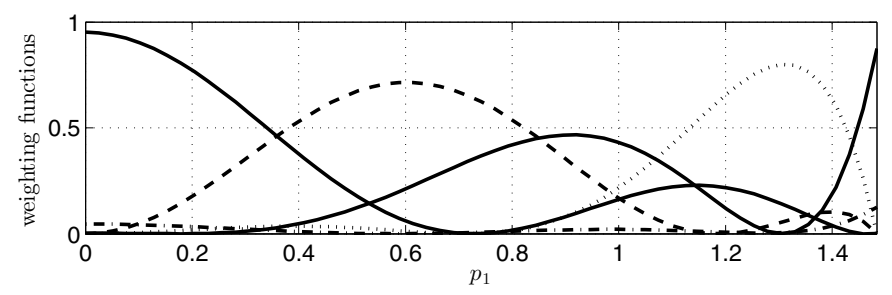

(c) Weighting functions of the CNO convex hull $\left(V_{C N O}=0.0827\right)$

Fig. 4. Results of the $\mathrm{CNO}$ convex hull manipulation for Example 2

average. Table I contains the actual numerical results of the comparison.

Some executions of the CNO method resulted in 5-10\% smaller hull than the constrained MVS case, because the $\mathrm{CNO}$ cost function does not strictly forces the vertices to the endpoints. Actually, the MVSA algorithm also has a tendency to stop in a local minimum considering that in certain cases (e.g., sphere-like shapes) there is no unique global minimum.

\section{CONCLUSION}

In this paper, we introduced a novel approach incorporated in the TP Model Transformation for tight convex hull generation of polytopic LPV/qLPV system models.

The proposed method is based on the Minimal Volume Symplex Analysis and accordingly, the resulted convex hull is called MVS-type hull. The MVS algorithm shows significant advantages compared to the previously known approaches: It provides tighter hull than the widely used $\mathrm{CNO}$ method while the computation is much faster, even by a factor of 500 in practically relevant cases. Furthermore, the MVS method consistently leads to identical result in contrast to the random search-based $\mathrm{CNO}$, which is not repeatable in most cases. The results are supported by numerical examples, which show that the method gives a powerful solution for tight convex hull generation in systems and control applications.

\section{REFERENCES}

[1] P. Baranyi, Y. Yam, and P. Várlaki, Tensor Product Model Transformation in Polytopic Model-Based Control, ser. Automation and Control Engineering. Taylor \& Francis Group, 2013.

[2] R.-E. Precup and H. Hellendoorn, "A survey on industrial applications of fuzzy control," Computers in Industry, vol. 62, no. 3, pp. 213 - 226, 2011.

[3] S. Nagy, Z. Petres, and P. Baranyi, "TP Tool-a Matlab toolbox for TP model transformation," in Proc. of 8th International Symposium of Hungarian Researchers on Computational Intelligence and Informatics, 2007, pp. 483-495.

[4] J. A. Nelder and R. Mead, "A simplex method for function minimization," Computer journal, vol. 7, no. 4, pp. 308-313, 1965.

[5] J. M. Bioucas-Dias, A. Plaza, N. Dobigeon, M. Parente, Q. Du, P. Gader, and J. Chanussot, "Hyperspectral unmixing overview: Geometrical, statistical, and sparse regression-based approaches," Selected Topics in Applied Earth Observations and Remote Sensing, IEEE Journal of, vol. 5, no. 2, pp. 354-379, 2012.

[6] A. Zymnis, S. Kim, J. Skaf, M. Parente, and S. Boyd, "Hyperspectral image unmixing via alternating projected subgradients," in Signals, Systems and Computers, 2007. ACSSC 2007. Conference Record of the Forty-First Asilomar Conference on, Nov 2007, pp. 1164-1168.

[7] C.-Y. Chi, "Non-negative least-correlated component analysis for separation of dependent sources," in invited talk at the Workshop on Optimization and Signal Processing, The Chinese University Hong Kong, Hong Kong, 2007.

[8] X. Tao, B. Wang, L. Zhang, and J. Q. Zhang, "A new scheme for decomposition of mixed pixels based on nonnegative matrix factorization," in Geoscience and Remote Sensing Symposium, 2007. IGARSS 2007. IEEE International, July 2007, pp. 1759-1762.

[9] J. Li and J. M. Bioucas-Dias, "Minimum volume simplex analysis: a fast algorithm to unmix hyperspectral data," in Geoscience and Remote Sensing Symposium, 2008. IGARSS 2008. IEEE International, vol. 3. IEEE, 2008, pp. III-250.

[10] T.-H. Chan, C.-Y. Chi, Y.-M. Huang, and W.-K. Ma, "A convex analysisbased minimum-volume enclosing simplex algorithm for hyperspectral unmixing," IEEE Transactions on Signal Processing, vol. 57, no. 11, pp. 4418-4432, Nov. 2009.

[11] A. Agathos, J. Li, D. Petcu, and A. Plaza, "Multi-GPU implementation of the minimum volume simplex analysis algorithm for hyperspectral unmixing," IEEE Journal of Selected Topics in Applied Earth Observations and Remote Sensing, 2014, accepted.

[12] P. Baranyi, L. Szeidl, and P. Varlaki, "Numerical reconstruction of the HOSVD based canonical form of polytopic dynamic models," in Intelligent Engineering Systems, 2006. INES'06. Proceedings. International Conference on, 2006, pp. 196-201.

[13] P. Baranyi, "TP model transformation as a way to LMI-Based controller design," IEEE Transactions on Industrial Electronics, vol. 51, no. 2, pp. 387-400, Apr. 2004.

[14] L. De Lathauwer, B. De Moor, and J. Vandewalle, "A multilinear singular value decomposition," SIAM Journal on Matrix Analysis and Applications, vol. 21, no. 4, pp. 1253-1278, 2000.

[15] P. Baranyi, "Convex hull generation methods for polytopic representations of LPV models," in Applied Machine Intelligence and Informatics, 2009. SAMI 2009. 7th International Symposium on, Jan 2009, pp. 69-74.

[16] P. Várkonyi, D. Tikk, P. Korondi, and P. Baranyi, "A new algorithm for RNO-INO type tensor product model representation," in 2005 IEEE International Conference on Intelligent Engineering Systems, INES, vol. 5, 2005, pp. 263-266.

[17] J. Nascimento and J. Dias, "Vertex component analysis: a fast algorithm to unmix hyperspectral data," IEEE Transactions on Geoscience and Remote Sensing, vol. 43, no. 4, pp. 898-910, Apr. 2005.

[18] S. P. Boyd, Linear matrix inequalities in system and control theory. Philadelphia: Society for Industrial and Applied Mathematics, 1994. 